\title{
New Sequence-Defined Polyaminoamides with Tailored Endosomolytic Properties for Plasmid DNA Delivery
}

\author{
David Schaffert, ${ }^{*}{ }^{\dagger}, \#$ Christina Troiber, ${ }^{\dagger}$ and Ernst Wagner ${ }^{*}{ }^{\dagger}$ \\ ${ }^{\dagger}$ Pharmaceutical Biotechnology, Department of Pharmacy, Center for Drug Research, and ${ }^{\ddagger}$ Center for NanoScience (CeNS), \\ Ludwig-Maximilians-Universität Munich, Germany
}

\section{Supporting Information}

ABSTRACT: Heterogeneity of polymeric carriers is one of the most elusive obstacles in the development of nonviral gene delivery systems, concealing interaction mechanisms and limiting the use of structureactivity relationship studies. In this report, novel sequence-defined polyaminoamides, prepared by solid-phase assisted synthesis, were used to establish first structure-activity relationships for polymerbased plasmid DNA delivery. By combining a cationic building block with hydrophobic modifications and bioreversible disulfide crosslinking sites, transfection polymers with tailored lytic and DNA binding properties were designed. These polymers demonstrated clear correlation between structure and performance in lysis and DNA binding assays. In vitro studies showed negligible toxicity and highly efficient gene transfer, demonstrating the potential of this platform in the fast, combinatorial development of new transfection polymers.

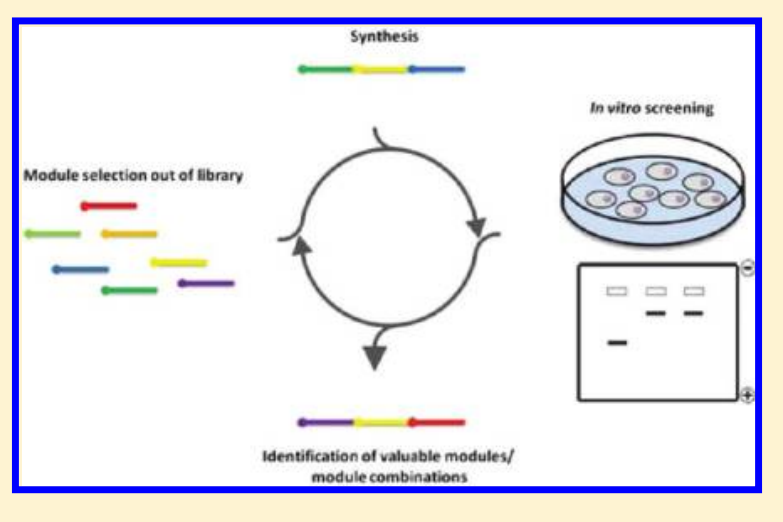

\section{INTRODUCTION}

Gene therapy is a promising therapeutic strategy based on the concept of treating a disease at its molecular point of origin. ${ }^{1}$ By introducing new genetic material into target cells, deficient genes can be replaced by functional copies, new genes can be introduced into the cell, or existing expression patterns can be altered. These properties make gene therapy a potentially useful tool for treating diseases, which cannot be remedied by more conventional means. $^{2-4}$ Broad clinical use has not been achieved so far, due to the specific hurdles of delivering nucleic acids (NA) to the target cells. While recombinant viral delivery vectors are quite effectively used in clinical studies and demonstrate unequaled delivery efficiency, there are major concerns about their clinical safety with special emphasis on risks like insertional mutagenesis, immunogenicity, and inflammatory potential. ${ }^{5,6}$ Polymeric delivery systems as alternatives allow faster development circles, easy modification, and increased loading capacity, and are in general better tolerated by the immune system. However, despite significant progress in the development of polymeric carriers, they are still characterized by a rather low efficiency and their potential is far from being fully exploited.

The low efficiency of nonviral vectors stems from the numerous extracellular and intracellular barriers entrapping or destroying significant amounts of the payload before entering the cell. ${ }^{7}$ As most of the carrier systems enter cells by endosomal uptake routes, one of the biggest barriers is the successful escape out of the endosome. Viral delivery systems use a sophisticated protein machinery to interact with the endosomal microenvironment with the aim to escape the endosome. ${ }^{8}$ The hemagglutinin, a glycoprotein of influenza virus $\mathrm{A}$, for example, is triggered by the acidification of the endosome, causing a conformational change, which results in the fusion between viral and host cell membranes and penetration of the viral genome into the cytosol. ${ }^{9}$ Polymeric systems employing complex structural changes like this have not been constructed up to now, but the described systems exploit similar endosomal escape characteristics. Polymeric carriers like polyethylenimine (PEI) or polyamidoamine (PAMAM) dendrimers utilize their high intrinsic buffer capacity to cause an osmotic burst of the endosome. ${ }^{10}$ The required high endosomal concentration of these polymers necessitates the use of a large excess or a high $M_{\mathrm{w}}$ species to achieve an acceptable rate of success. To further improve their efficiencies, different strategies were introduced, e.g., the incorporation of membrane disrupting peptides ${ }^{11,12}$ into the delivery system, or the use of hydrophobic modifications. These modifications increase the interactions with cellular membranes $^{13,14}$ and have also been used for stabilization and modulation of polymer nanoaggregate formation in vitro and in vitro. ${ }^{15,16}$ Although all of these approaches have their advantages, they are still limited by the inherent heterogeneity of the polymeric carriers, which conceals precise structureactivity relationships. This is especially important for programmed, bioresponsive delivery systems, which consist of multiple domains including elements for targeting, shielding,

Received: November 14, 2011

Revised: April 16, 2012

Published: May 2, 2012 
and release of the payload. ${ }^{17}$ Increased molecular precision in the assembly of these systems would allow the design of even more complex systems. Hartman et al. ${ }^{18}$ demonstrated recently the synthesis of linear polyaminoamides (PAAs) using solidphase assisted synthesis. Adopting this approach for combinatorial polymer development, although with a different chemical strategy, gave access to highly defined and functionalized polymers. We recently described the synthesis of protected polyamino acid building blocks for fmoc solid-phase assisted polymer synthesis, ${ }^{19}$ and their application in the construction of a modular polymeric platform for plasmid DNA (pDNA) and siRNA delivery was reported in a short communication. ${ }^{20}$ The current paper describes in more detail the design and evaluation of 26 modular constructed PAAs for pDNA delivery. Polymers with tailored complexation capabilities and lytic activity were constructed by combining different elements for DNA binding, $\mathrm{pH}$-triggered endosomal escape, and bioresponsive stabilization. Correlation of the results of $\mathrm{pDNA}$ complexation and $\mathrm{pH}$ dependent lytic activity assays with their in vitro pDNA transfection potential allowed establishment of first structureactivity relationships for this new class of transfection reagents.

\section{MATERIALS AND METHODS}

Materials. Fmoc-amino acids and resins (base resins and preloaded resins) were purchased from IRIS Biotech, Marktredwitz, and Novabiochem GmbH, Darmstadt. PyBOP was bought from MultiSynTech GmbH, Witten. DCM, DMF, $\mathrm{MeOH}$, and THF were obtained from Merck and distilled before use. Water was used as purified, deionized water. LPEI and fmoc-Stp(boc) $)_{3}-\mathrm{OH}$ were synthesized as described. ${ }^{19,21}$ DMSO, EtOH, ACN, deuterated solvents, and MTT bromide were obtained from Sigma-Aldrich (Munich, Germany). Plasmid pEGFPLuc (encoding a fusion of enhanced green fluorescent protein (EGFP) and Photinus pyralis luciferase under control of the CMV promoter) was produced with the Qiagen Plasmid Giga Kit (Qiagen, Hilden, Germany) according to the manufacturer recommendations. Cell culture media, antibiotics, and fetal calf serum were purchased from Invitrogen (Karlsruhe, Germany). Luciferase cell culture lysis buffer and Dluciferin sodium salt were obtained from Promega (Mannheim, Germany). All polymer characterization data can be found in the Supporting Information.

Synthesis of i-Shapes with two FAs: HO-K-Stp $1-K-\mathrm{FA}_{2}$. After swelling of fmoc-Lys(boc)-Wang resin (0.05-0.20 mmol) in DCM (30 $\mathrm{min})$ and cleavage of the fmoc protecting group (20\% piperidine in DMF, twice), 4 equiv of a solution of fmoc$\mathrm{Stp}\left(\right.$ boc) ${ }_{3}-\mathrm{OH}$ in DMF, DIPEA (8 equiv), and $\mathrm{PyBOP} / \mathrm{HOBt}$ (4 equiv) were added to the resin, and the vessel was agitated until Kaiser test indicated complete conversion $(30 \mathrm{~min})$. The reaction solvent was drained, and the resin was washed five times with DMF. To couple two fatty acids to the $\mathrm{N}$-terminus of the PAA, fmoc-Lys(fmoc)-OH was incorporated before the coupling of the fatty acid. To cap residual, unreacted primary amino groups before introduction of the fatty acid, the resin was acetylated using 5 equiv of acetic anhydride and 10 equiv of DIPEA, before subsequent removal of the fmoc protecting group. The resin was washed three times with DMF followed by three DCM washes after removal of the fmoc protecting group. Ten equivalents of the fatty acid were dissolved in DCM and were added to the resin together with 10 equiv of PyBOP/ HOBt and 20 equiv of DIPEA in the smallest possible amount of DMF. The mixture was agitated until Kaiser test indicated complete conversion (normally $30 \mathrm{~min}$ ). After completion of the reaction, the resin was washed with DMF, DCM, and nhexane and dried for $12 \mathrm{~h}$ over $\mathrm{KOH}$ in vacuo.

Synthesis of i-Shapes with Two Coupling Domains: $\mathrm{HO}-\mathrm{C}-\mathrm{Stp}_{3}-\mathrm{C}-\mathrm{K}-\mathrm{FA}_{2}$. After swelling $0.035 \mathrm{mmol}$ of a fmocCys(trt)-Wang resin in DCM and cleavage of the fmoc protecting group (20\% piperidine in DMF, twice), 4 equiv of a solution of fmoc-Stp (boc) ${ }_{3}-\mathrm{OH}$ in DMF, DIPEA (8 equiv), and $\mathrm{PyBOP} / \mathrm{HOBt}$ (4 equiv) were added to the resin, and the vessel was agitated until Kaiser test indicated complete conversion (normally $30 \mathrm{~min}$ ). The reaction solvent was drained and the resin was washed five times with DMF. This cycle was repeated twice. Afterward, the amino acid fmocCys(trt)-OH was coupled. Then, in order to couple two fatty acids to the linear PAA, fmoc-Lys(fmoc)-OH was incorporated at the $\mathrm{N}$-terminus before coupling the fatty acid. To cap unreacted primary amino groups, the resin was acetylated using 5 equiv of acetic anhydride and 10 equiv of DIPEA, before the subsequent removal of the fmoc protecting group. To couple the fatty acid, the solvent was changed to DCM after fmoc cleavage. Therefore, after removal of the fmoc protecting group, the resin was washed three times with DMF and DCM. Ten equivalents of the fatty acid were dissolved in DCM; 20 equiv of DIPEA, and 10 equiv of PyBOP/HOBt in DMF were added to the resin; and the mixture was agitated for $30 \mathrm{~min}$. After completion of the reaction, the resin was washed with DMF, $\mathrm{DCM}$, and $n$-hexanes and dried over $\mathrm{KOH}$ in vacuo.

Synthesis of T-Shapes with Two FAs: HO-C-Stp $1-K(\mathrm{~K}-$ $F_{2}$ )-Stp ${ }_{1}$-C. After swelling 0.05-0.20 mmol of fmoc-Cys(trt)Wang resin in DCM for $30 \mathrm{~min}$, the fmoc protection group was cleaved (20\% piperidine in DMF, twice). After washing the resin, 4 equiv of fmoc-Stp(boc) ${ }_{3}-\mathrm{OH}$, DIPEA (8 equiv) and PyBOP/HOBt (4 equiv) was added for $30 \mathrm{~min}$. The reaction solvent was drained and the resin was washed five times with DMF. Reaction progress was monitored by Kaiser test. To introduce a branching point, dde-Lys (fmoc)-OH was used in the next coupling step. Dde-Lys(fmoc)-OH (4 equiv), DIPEA (8 equiv), and PyBOP/HOBt (4 equiv) dissolved in DMF were added, and the synthesis vessel was agitated for $30 \mathrm{~min}$. After a negative Kaiser test, the resin was washed with DMF. After treatment with $20 \%$ piperidine in DMF and washing the resin with DMF, fmoc-Lys(fmoc)-OH (4 equiv), DIPEA (8 equiv), and PyBOP)/HOBt (4 equiv) were added. In order to cap unreacted primary amino groups, the resin was acetylated using 5 equiv of acetic anhydride and 10 equiv of DIPEA, before the subsequent removal of the fmoc protecting group. To couple the fatty acid, the solvent was changed to DCM after fmoccleavage. Therefore, the resin was washed three times with DMF and DCM after removal of the fmoc protecting group. Ten equivalents of the fatty acid dissolved in the minimal amount of DCM, 20 equiv of DIPEA and 10 equiv of PyBOP/ $\mathrm{HOBt}$ were added to the resin for $30 \mathrm{~min}$. After completion of the reaction, the resin was washed five times with DCM and three times with DMF. The dde protecting group was cleaved using $2 \%$ hydrazine monohydrate in DMF (10-15 times for 5 min) until no significant $A_{300}$ was measurable in the deprotection mixture. After washing the resin 5 times with DMF, fmoc-Stp(boc) ${ }_{3}$-OH, DIPEA (8 equiv) and PyBOP/ HOBt (4 equiv) in DMF were added for $30 \mathrm{~min}$. After successful reaction, the resin was treated twice with $20 \%$ piperidine in DMF. After washing the resin, boc-Cys(trt)-OH (4 equiv) dissolved in DMF, DIPEA (8 equiv), and PyBOP/ HOBt (4 equiv) were added for $30 \mathrm{~min}$. Afterward, the resin was washed and dried over $\mathrm{KOH}$ in vacuo. 
Cleavage Conditions. For cleavage, the resin was treated with $10 \mathrm{~mL} / \mathrm{g}_{(\mathrm{resin})}$ of cleavage solution: PAAs without cysteines were treated with a TFA/water (95:5) mixture, whereas cysteine-containing PAAs were treated with a TFA/Water/ TIS (95:2.5:2.5) mixture for $1-2 \mathrm{~h}$. The resin was filtered off and washed twice using pure TFA followed by one DCM wash. The combined filtrates were concentrated and either precipitated by dropwise addition into ice-cold MTBE or other suitable mixtures. The precipitate was collected by centrifugation. The precipitate/film was dissolved in 5\% acetic acid, snap-frozen, and lyophilized to obtain the PAA.

DNA Polyplex Formation Using PAA-Based Carriers. Polyplex formulations for pDNA delivery were prepared as follows: $200 \mathrm{ng}$ of $\mathrm{pDNA} /$ well and the calculated amount of PAA were diluted in separate tubes in HBG pH 7.4. The pDNA solution was added to the polymer solution, mixed by pipetting, and incubated for $30-40 \mathrm{~min}$ at $\mathrm{RT}$ in order to form the polyplexes.

Electrophoretic Mobility Shift Assay. A 1\% agarose gel was prepared by dissolving $1.2 \mathrm{~g}$ agarose in $120 \mathrm{~mL}$ TBE buffer and heating the mixture to $100{ }^{\circ} \mathrm{C}$. After cooling down to approximately $50{ }^{\circ} \mathrm{C}, 120 \mu \mathrm{L}$ Gel-Red $(1 \mathrm{mg} / \mathrm{mL})$ were added and the gel was poured in a casting unit. Polyplex-samples containing $200 \mathrm{ng}$ pDNA, polymer, HBG-buffer, and loading buffer were placed into the pockets after an incubation time of $30 \mathrm{~min}$ at RT. Electrophoresis was performed at $120 \mathrm{~V}$ for 80 min.

Erythrocyte Leakage Assay. Murine erythrocytes were isolated from fresh, citrate buffered blood and washed with phosphate-buffered saline (PBS) several times. The erythrocyte pellet was diluted to $5 \times 10^{7}$ erythrocytes per $\mathrm{mL}$ with PBS ( $\mathrm{pH} 7.4,6.5$, and 5.5). The polymers were diluted in $75 \mu \mathrm{L}$ of PBS in a V-bottom 96-well plate (NUNC, Denmark). For $100 \%$ lysis, control wells contained buffer with $1 \%$ Triton X100. A volume of $75 \mu \mathrm{L}$ of erythrocyte suspension was added to each well, and the plates were incubated at $37{ }^{\circ} \mathrm{C}$ under constant shaking for $1 \mathrm{~h}$. After centrifugation, $80 \mu \mathrm{L}$ of the supernatant was analyzed for hemoglobin release at $405 \mathrm{~nm}$ using a microplate plate reader (Spectrafluor Plus, Tecan Austria $\mathrm{GmbH}$, Grödig, Austria). PBS-buffers with $\mathrm{pH}$ values of $7.4,6.5$, and 5.5 were used as negative control. Relative hemolysis was defined as hemolysis $(\%)=\left(A_{405}\right.$ (PAA treated $)$ - $A_{405}($ buffer treated $\left.)\right) /\left(A_{405}\left(\right.\right.$ TritonX treated - $\left(A_{405}(\right.$ Buffer treated)) $\times 100$.

Measurement of Particle Size via Dynamic Light Scattering. DNA polyplexes were formed as follows: $10 \mu \mathrm{g}$ of pEGFPLuc and $100 \mu \mathrm{g}$ or $200 \mu \mathrm{g}$ polymer, resulting in a weight to weight of 10 or 20 , respectively, were diluted in separate tubes in buffer ( $20 \mathrm{mM}$ HEPES $\mathrm{pH} 7.4)$ to a total volume of $25 \mu \mathrm{L}$. The pDNA solution was added to the polymer solution and mixed by rapidly pipetting up and down at least 5 times, resulting in a final pDNA concentration of 200 $\mu \mathrm{g} / \mathrm{mL}$. The incubation time was $30-40 \mathrm{~min}$, in order to complete polyplex formation. The polyplex solution was then diluted 1:20 with buffer and measured in a folded capillary cell (DTS1061) with laser light scattering using a Zetasizer Nano ZS with backscatter detection (Malvern Instruments, Worcestershire, UK).

Luciferase Reporter Gene Expression. Cells were plated in 96 -well plates at a density of 10000 cells/well $24 \mathrm{~h}$ prior to transfection. The polyplexes, formed using $200 \mathrm{ng}$ of pDNA/ well, were added to the cells in $100 \mu \mathrm{L}$ culture medium containing $10 \%$ serum, $100 \mathrm{U} / \mathrm{mL}$ penicillin, and $100 \mu \mathrm{g} / \mathrm{mL}$ streptomycin. Twenty-four hours after initial transfection, medium was removed and cells were lysed in $50 \mu \mathrm{L} 0.5 \times$ Promega cell lysis solution to measure the gene expression. Luciferase activity was measured using a Lumat LB9507 instrument (Berthold, Bad Wildbad, Germany). Luciferase light units were recorded from a $20 \mu \mathrm{L}$ aliquot of the cell lysate with $10 \mathrm{~s}$ integration time after automatic injection of freshly prepared luciferin using the luciferase assay system (LAR, Promega, Mannheim, Germany). Transfection efficiency was evaluated as relative light units (RLU) per number of seeded cells. Two nanograms of recombinant luciferase (Promega, Mannheim, Germany) corresponded to $10^{7}$ light units.

Cell Viability Assay. The metabolic activity of polyplextreated cells was determined in parallel to the transfection studies using a methylthiazole tetrazolium (MTT) $)^{22}$ assay. Twenty-two hours after initiation of transfection, $10 \mu \mathrm{L}$ per 100 $\mu \mathrm{L}$ of medium of a $5 \mathrm{mg} / \mathrm{mL}$ solution of MTT in sterile PBSbuffer was added per well. After incubation for $1-2 \mathrm{~h}$ at $37^{\circ} \mathrm{C}$, the medium was removed and the cells were frozen at $-80{ }^{\circ} \mathrm{C}$ for at least 1 h. $200 \mu \mathrm{L}$ DMSO were added and the samples were incubated under constant shaking at $37^{\circ} \mathrm{C}$ for $30 \mathrm{~min}$ to dissolve the crystals completely. The optical absorbance was measured at $590 \mathrm{~nm}$ with a reference wavelength of $630 \mathrm{~nm}$ using a microplate reader (Spectrafluor Plus,Tecan Autstria $\mathrm{GmbH}$, Grödig, Austria). The relative cell viability was defined as viability $(\%)=A_{590}($ polyplex treated $) / A_{590}($ Buffer treated control) $\times 100$.

\section{RESULTS AND DISCUSSION}

Design and Synthesis of Sequence-Defined Transfection Polymers. A small library of sequence-defined PAAs was designed with the aim of identifying a set of minimal prerequisites for efficient pDNA delivery. The design was limited to three structural motifs: compaction domain, lipophilic element, and dimerizing/cross-linking anchors. The compaction domain was introduced by variable repeats of Stp, an artificial polyamino acid mimicking the diaminoethane motif of PEI. ${ }^{19}$ Lipophilic chains of varying length $\left(\mathrm{C}_{4}, \mathrm{C}_{8}, \mathrm{C}_{14}, \mathrm{C}_{18}\right)$, anchored to the polymers either via their $\mathrm{N}$-terminus or by acylation of both amines of a lysine, were used to control the lipophilicity. Cysteines were introduced to compensate for the rather small size of the synthesized polymers $\left(M_{\mathrm{w}} \leq 3100 \mathrm{Da}\right)$ and to limit possible polyplex destabilization in serum. The disulfide bonds formed by oxidation of cysteine thiols have been reported to stabilize polyplexes in the rather low extracellular reductive potential and can be reduced in the cytosol to facilitate pDNA release. ${ }^{23}$

Figure 1 shows the general structures of the synthesized polymer families. The exact configuration of the modules was varied in the different families to allow impact analysis of the different modules. The simplest structural family is the PAA chain (structure I), constructed by linear chain elongation using Stp and/or amino acid units. These polymers, comparable to OEI800 in $M_{\mathrm{w}}$ and number of protonable amines, were included as negative controls. The PAA chain (structure II) contains two cysteine residues, introducing the ability to form disulfide bridges. Both families were hydrophobically modified using fatty acids (FA) at their N-terminus, resulting in the i-shape families (structures III + IV). The dimerizing i-shapes (structure V) were synthesized to test polyplex stabilization using dimerization anchors, whereas the symmetrical T-shape family (structure VI) was used to examine the influence of a changed polymer topology on 


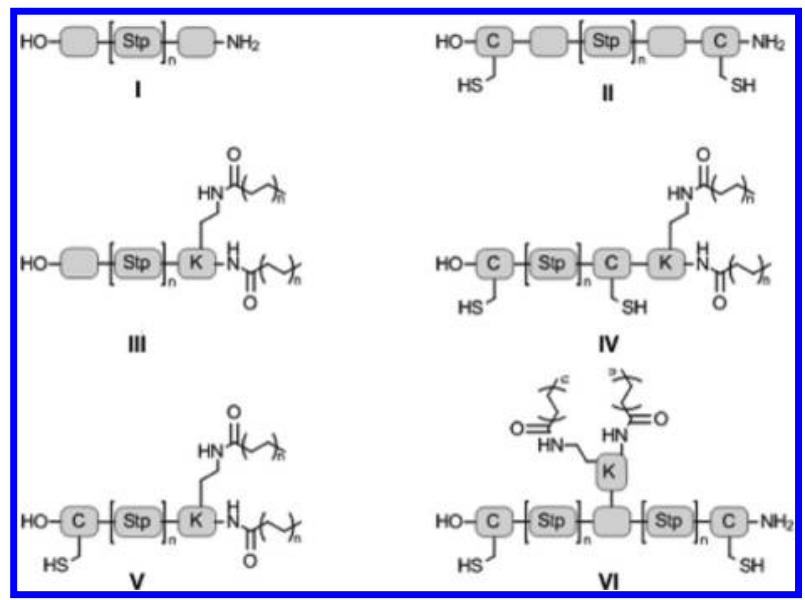

Figure 1. Structural overview over the different polymer families in the library. PAA chain (I), PAA chain with cross-linking cysteines (II), $\mathrm{PAA}$ with acylation at $\mathrm{N}$-terminal lysine (i-shape type) without (III), with one dimerizing (V), or with two cross-linking cysteines (IV); PAA with acylation in the center ( $\mathrm{T}$-shape type) and cross-linking cysteines (VI).

transfection efficacy. Scheme 1 shows the general synthetic procedure using standard fmoc-synthesis conditions. A branching point was incorporated by using dde-Lys(fmoc)$\mathrm{OH}$ to introduce a bis-fatty acid modified lysine at the center of the molecule. After successful side chain deprotection and introduction of the fatty acid residues, the dde protective group on the $\alpha$-amino function was removed by hydrazine treatment and the synthesis continued. All polymers were cleaved from the support using a mixture of TFA $/ \mathrm{H}_{2} \mathrm{O}$ or TFA/ $\mathrm{H}_{2} \mathrm{O} / \mathrm{TIS}$ and characterized by ${ }^{1} \mathrm{H}$ NMR. The sequences of all used polymers can be found in Table 1.

Evaluation of pH-Dependent Lytic Activity. To escape endosomal entrapment, an effective lytic activity of the carrier is a desirable prerequisite for successful delivery of nucleic acids into the cytosol. Potential cytotoxic interactions with cell membranes in the beginning of the transfection process should be reduced by using $\mathrm{pH}$-specific lytic delivery systems, thereby
Table 1

\begin{tabular}{|c|c|}
\hline polymer & sequence $^{a}$ \\
\hline 1 & $\mathrm{~K}-\mathrm{Stp}_{1}-\mathrm{K}$ \\
\hline 6 & $\mathrm{~K}-\mathrm{Stp}_{2}-\mathrm{K}$ \\
\hline 23 & $\mathrm{~K}-\mathrm{Stp}_{5}-\mathrm{K}$ \\
\hline 34 & K-Stp -ButA $^{-}$ \\
\hline 35 & $\mathrm{~K}-\mathrm{Stp}_{2}$-CapA \\
\hline 36 & $\mathrm{~K}-\mathrm{Stp}_{2}-\mathrm{MyrA}$ \\
\hline 37 & $\mathrm{~K}-\mathrm{Stp}_{2}$-OleA \\
\hline 8 & K-Stp 2 -K-CapA $A_{2}$ \\
\hline 9 & $\mathrm{~K}-\mathrm{Stp}_{2}-\mathrm{K}-\mathrm{MyrA}_{2}$ \\
\hline 10 & K-Stp 2 -K-OleA 2 \\
\hline 21 & K-Stp $4-\mathrm{K}-\mathrm{MyrA}_{2}$ \\
\hline 22 & $\mathrm{~K}-\mathrm{Stp}_{4}-\mathrm{K}-\mathrm{OleA} \mathrm{A}_{2}$ \\
\hline 69 & C-Stp $2-\mathrm{K}-\mathrm{MyrA}{ }_{2}$ \\
\hline 70 & C-K-Stp $2-\mathrm{K}-\mathrm{MyrA}_{2}$ \\
\hline 72 & C-Stp $2-\mathrm{K}-\mathrm{OleA}{ }_{2}$ \\
\hline 71 & C-K-Stp2-K-OleA ${ }_{2}$ \\
\hline 51 & $\mathrm{C}-\mathrm{Stp}_{3}-\mathrm{C}-\mathrm{K}$ \\
\hline 45 & C-Stp 3 -C-K-MyrA 2 \\
\hline 46 & $\mathrm{C}-\mathrm{Stp}_{3}$-C-K-OleA 2 \\
\hline 76 & $\mathrm{C}-\mathrm{Stp}_{1}-\mathrm{K}(\mathrm{K}-\mathrm{OleA} 2)-\mathrm{Stp}_{1}-\mathrm{C}$ \\
\hline 49 & C-Stp $2-\mathrm{K}-(\mathrm{K}-\mathrm{OleA} 2)-\mathrm{Stp}_{2}-\mathrm{C}$ \\
\hline 80 & $\mathrm{C}-\mathrm{Stp}_{3}-\mathrm{K}(\mathrm{K}-\mathrm{OleA} 2)-\mathrm{Stp}_{3}-\mathrm{C}$ \\
\hline 84 & $\mathrm{C}-\mathrm{Stp}_{4}-\mathrm{K}(\mathrm{K}-\mathrm{OleA} 2)-\mathrm{Stp}_{4}-\mathrm{C}$ \\
\hline 74 & C-Stp ${ }_{1}-\mathrm{K}(\mathrm{K})-\mathrm{Stp}_{1}-\mathrm{C}$ \\
\hline 78 & $\mathrm{C}-\mathrm{Stp}_{3}-\mathrm{K}(\mathrm{K})-\mathrm{Stp}_{3}-\mathrm{C}$ \\
\hline 82 & $\mathrm{C}-\mathrm{Stp}_{4}-\mathrm{K}(\mathrm{K})-\mathrm{Stp}_{4}-\mathrm{C}$ \\
\hline
\end{tabular}

${ }^{a}$ ButA, Butyric acid; CapA, Caprylic acid; MyrA, Myristic acid; OleA, Oleic acid.

lowering the overall cytotoxic potential of the carrier. The different polymer families were screened in a mouse erythrocyte leakage assay system to identify structural motifs, resulting in lytic activity. All measured values were buffercorrected and lysis caused by $1 \%$ Triton X-100 was set as $100 \%$. By testing the polymers at different $\mathrm{pH}$ values, comparable to the $\mathrm{pH}$ of different stages of the endosome, ${ }^{24}$ highly $\mathrm{pH}$-specific lytic polymers were identified. Figure 2a shows the lytic activity

Scheme 1. Solid-Phase Synthesis Procedure Exemplified by T-Shape Synthesis

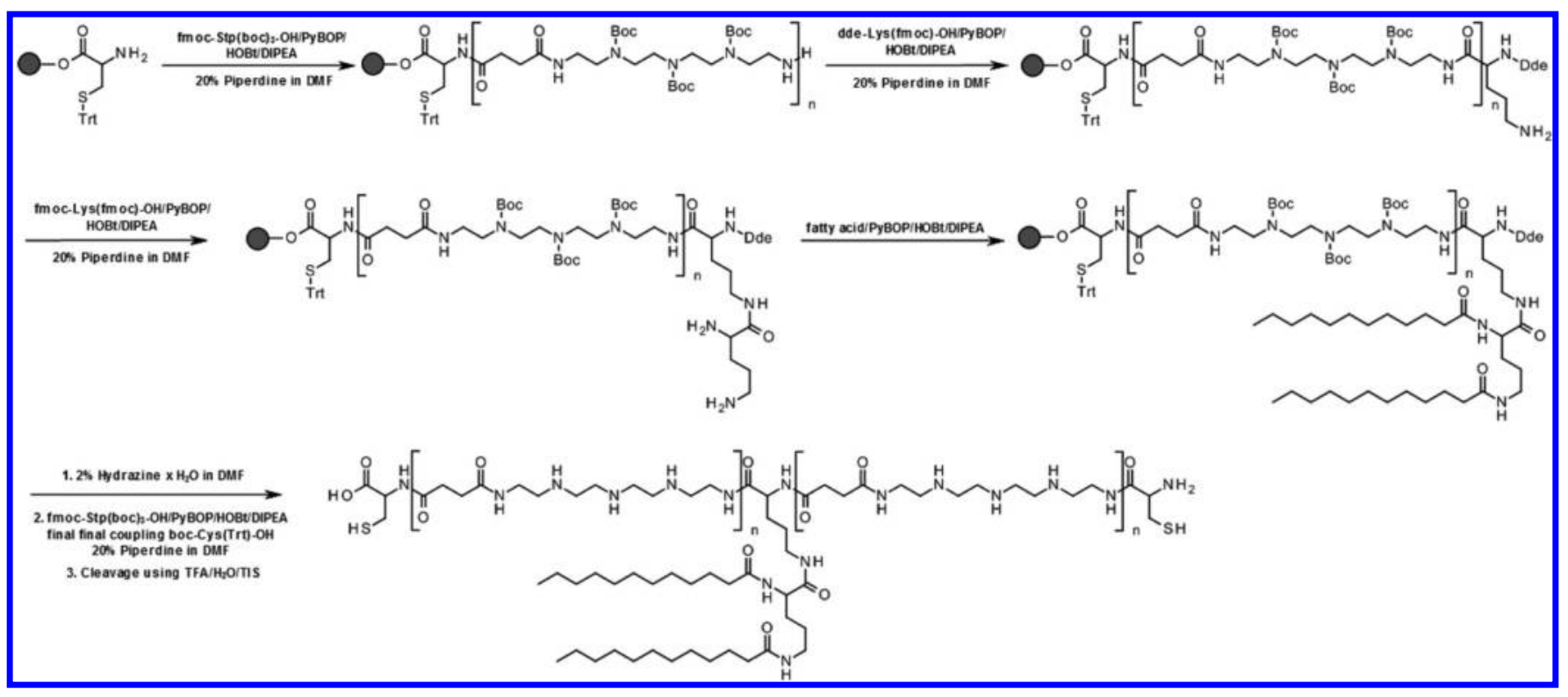




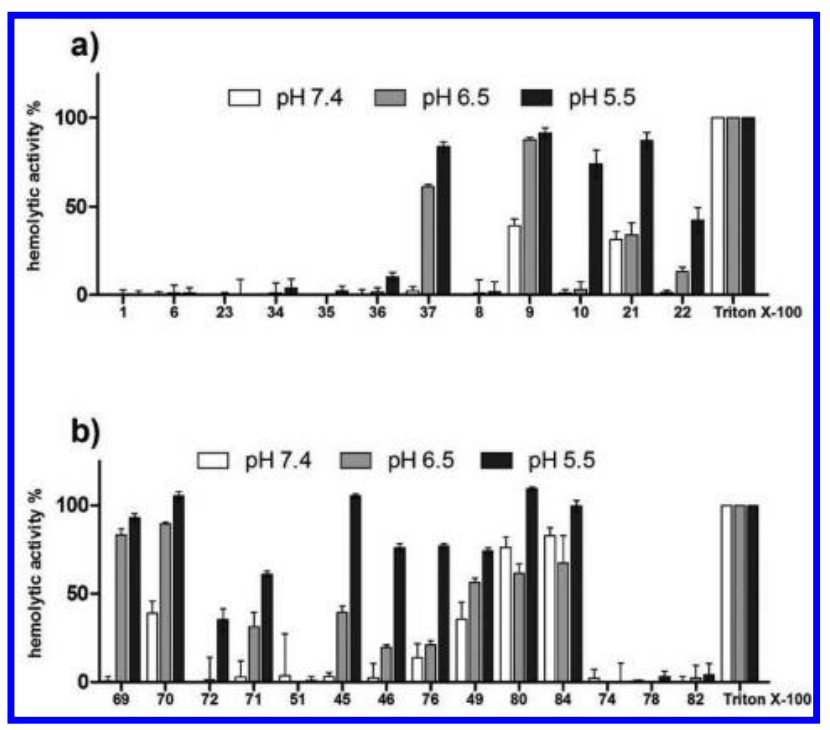

Figure 2. Relative $\mathrm{pH}$-dependent lytic activity of the polymer library $(5 \mu \mathrm{M})$. All values are buffer corrected; $1 \%$ Triton-X 100 was used as positive control. (a) Non-thiol-containing polymers. (b) Thiolcontaining polymers.

of a subset of the library at $\mathrm{pH} 5.5, \mathrm{pH} 6.5$, and $\mathrm{pH} 7.4$ in PBS. Lytic activity of the unmodified PAA backbone was assessed by the model sequences K-Stp 1 -K (1), K-Stp 2 -K (6); K-Stp ${ }_{5}-\mathrm{K}$ (23). These plain PAA sequences did not demonstrate any lytic activity even at concentrations $>5 \mu \mathrm{M}$ (data not shown). Introduction of a single N-terminal fatty acid into a K-Stp 2 sequence (34-37) showed a slight increase in activity with oleic (37) and myristic acid (36) as the most effective modifications. In contrast, polymers modified with fatty acids < $\mathrm{C}_{14}(34,35)$ were not lytic.

Lytic potency of the polymers was further increased by using a N-terminal lysine as branching point for two fatty acids (double fatty acid motif). The increased lytic activity can be attributed to the close vicinity of the fatty acids resembling the general structure of amphipathic lipids. ${ }^{25}$ Acylation of the Nterminal lysine, using caprylic acid (8), did not result in strong lytic activity, due to the rather short alkyl chain. Incorporation of myristyl residues (9), however, resulted in strong, unspecific lysis already causing up to $40 \%$ of erythrocyte rupture at neutral $\mathrm{pH}$. Oleic acid modification (10) led to moderate, highly $\mathrm{pH}$ specific lytic activity, rendering this modification the most valuable. A generally advantageous trend of an increased activity at lower $\mathrm{pH}$ was observed for all PAAs double-modified with fatty acid. This $\mathrm{pH}$-specificity might be caused by the increased protonation of the polyamine backbone at the slightly acidic $\mathrm{pH}$ and, thus, increased interaction with negatively charged domains of the cell membranes. The size of the nucleic acid binding domain had only moderate effects on lytic activity (21 vs 9,10 vs 22) and did not influence the $\mathrm{pH}$-dependency. A plausible explanation is the reduced molar proportion of the hydrophobic domain in the polymer, indicating that 2-3 Stp units may be the optimal chain length for specific and high lytic activity, using polymers with one hydrophobic module. The introduction of cysteines into the sequence as dimerization/ cross-linking handle did not significantly alter the extent and $\mathrm{pH}$-specificity of erythrocyte lysis $(9,10$ vs 70,71 and 45,46$)$.

The position of the hydrophobic element had no significant influence on the specificity of lytic activity, when comparing oleic acid modified i-shapes and T-shapes (45 vs 76 ). However, with increasing molecular weight of the T-shapes $(76,49,80$, 84) an increase in general lytic potency was observed. This is probably connected to a stronger interaction of the polymers with the negatively charged cell membrane. The corresponding control polymers $(\mathbf{7 4}, \mathbf{7 8}, \mathbf{8 2})$ without fatty acid modification demonstrated no lytic activity, confirming the need of hydrophobic modification for lytic cell membrane interaction.

The results clearly demonstrate that the lytic activity of the polymers can be controlled by their molecular structure, especially by the substitution pattern and type of fatty acids used. Whereas unmodified PAAs demonstrated no lytic activity, introduction of fatty acids with a chain length of $>\mathrm{C}_{8}$ resulted in significant lytic activity. This was amplified by the use of a double fatty acid motif. The $\mathrm{pH}$-specificity of the lytic activity was controlled by the type of fatty acid used. Oleic acid modification resulted in the most specific lytic activity of the tested polymers.

DNA Binding. The interaction of the polymers with pDNA was evaluated using an agarose gel shift assay (Figure 3 ), in

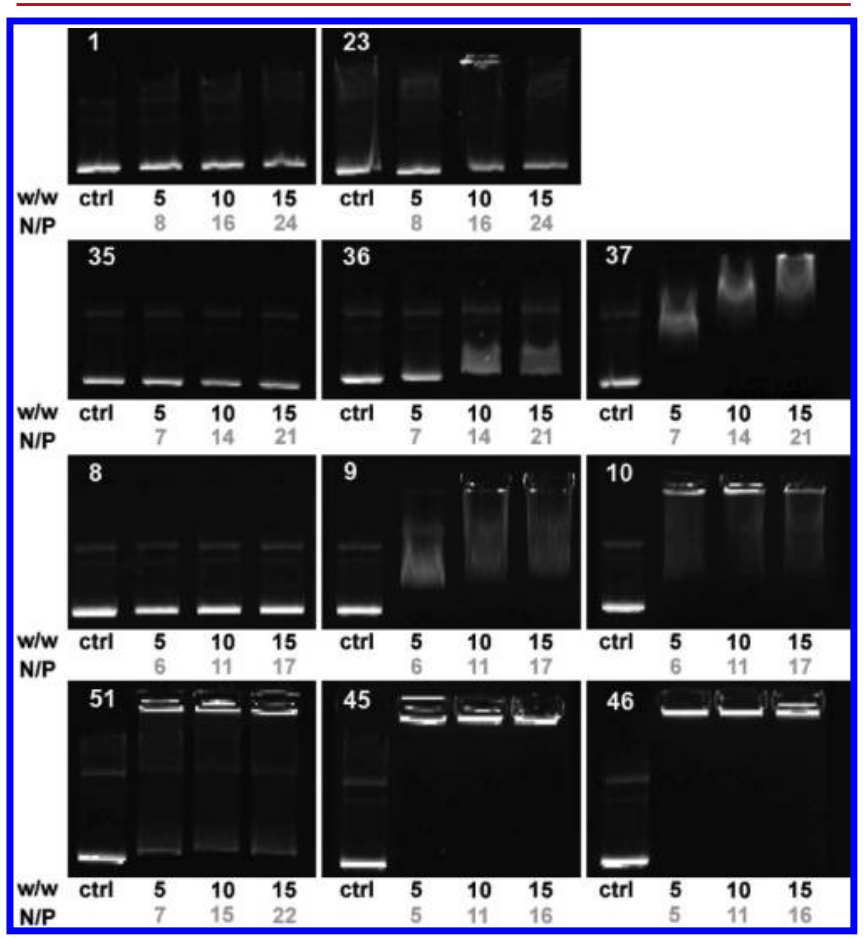

Figure 3. Electrophoretic mobility shift assay of different PAA/pDNA complexes in $\mathrm{HBG}$ at different $\mathrm{w} / \mathrm{w}$ ratios.

which binding strength correlates to the amount of polymer needed for complete pDNA retardation. Polymers with only Stp and terminal lysines were not able to inhibit pDNA migration at the tested concentrations $(1,23)$. To identify a

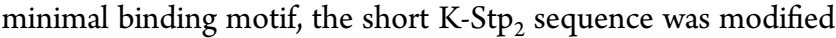
either with a single N-terminal fatty acid $(35-37)$ or the double fatty acid modified lysine motif $(\mathbf{8}-\mathbf{1 0})$, which were already used to increase the lytic activity. Single N-terminal fatty acid modification of the polymers resulted only in the case of oleic acid (37) in some, however, weak pDNA interaction at $\mathrm{w} / \mathrm{w} 15(\mathrm{~N} / \mathrm{P}=21)$. Introduction of a second fatty acid via a lysine branching point significantly improved interaction with pDNA. The dual fatty acid motif displayed strong pDNA/ polymer interactions with almost complete pDNA retardation at a w/w of $10(8-10, N / P=11)$ if the fatty acid was either $\mathrm{C}_{14}(9)$ or $\mathrm{C}_{18}(\mathbf{1 0})$. Use of a $\mathrm{C}_{8}(8)$ modification did not result 
in pDNA retardation at the tested concentrations. These results were reflected by DLS-measurements (Figure 4). Thus, neither

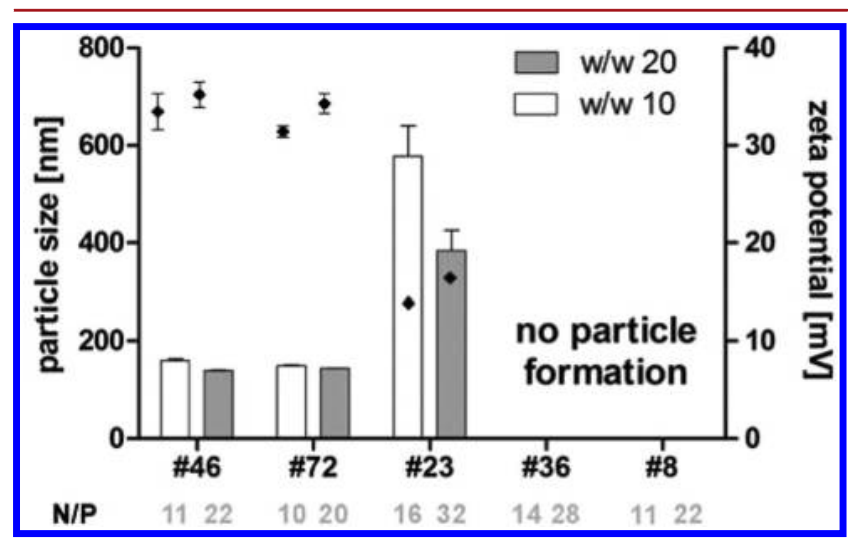

Figure 4. Particle size and zeta potential of selected examples out of the different PAA families, formed in HEPES buffer at different $\mathrm{w} / \mathrm{w}$ ratios (bars represent the measured particle size, rhombi the measured zeta potential).

the double $\mathrm{C}_{8}(8)$ modified nor the single $\mathrm{C}_{14}(36)$ modified polymer were able to form stable polyplexes. However, particle formation was observed with unmodified polymer 23 (17 protonable nitrogens). These polyplexes were characterized by a size of more than $400 \mathrm{~nm}$ and a zeta potential of $+15 \mathrm{mV}$, supporting the hypothesis of rather weak interactions between polymer and pDNA, as observed in the gel-shift assay. The introduction of two cysteines into the polymers $(45,46,51)$ showed only a moderate effect on polyplex stabilization in the gel shift assay. Polymer 51 containing 3 Stp units and 2 cysteines displayed incomplete pDNA retardation, whereas the fatty acid modified dimerizing polymers $(45,46)$ were able to efficiently complex the pDNA at $\mathrm{w} / \mathrm{w}$ ratios of $5(\mathrm{~N} / \mathrm{P}=5)$. Only polymers containing 6 or more Stp units and cysteine modifications (78, 82, Supporting Information) showed comparable interaction with pDNA. The stabilizing effect of cysteine incorporation could also be observed in DLS measurements (Figure 4; 46, 72). The resulting polyplexes had a size below $200 \mathrm{~nm}$ and a zeta potential around $+30 \mathrm{mV}$, irrespective of modification with one (72) or two cysteines (46).

Taken together, these results showed that polyplex stabilization was controlled by all three used motifs. Hydrophobic modification played a key role in lytic activity, but it had only supporting function in stabilizing the polyplex. By contrast, size of compaction domain (number of charges) and stabilization by disulfide bridging or cross-linking had a more pronounced influence on particle formation. This allowed tailoring of polymer/DNA interaction strength, which supported the formation of stable particles in the extracellular environment and their efficient disassembly in the cytosol.

Transfection Efficiency and Cytotoxicity. The transfection efficiency of the different polymers was assessed in vitro, using Neuro2A cells and employing a pEGFPLuc reporter gene system. Linear polyethylenimine $22 \mathrm{kDa}$ (LPEI $22 \mathrm{kDa}$ ) was used as control at its optimal $\mathrm{w} / \mathrm{w}$ ratio of $0.8(\mathrm{~N} / \mathrm{P}=6)$ to allow comparison of the different polymers. Due to the small size $(1-3 \mathrm{kDa})$ of the screened polymers, property changes due to an altered polymer sequence were reflected in transfection efficiency and cytotoxicity, allowing quick assessment of the potential benefit of a modification.
To assess their delivery capability and toxicity, plain PAA chains and fatty acid modified sequences (i-shapes) were compared to $\mathrm{K}-S t p_{5}-\mathrm{K}(23)$. This sequence resembles $\mathrm{OEI}_{800}$, a rather weak performing member of the PEI family, in terms of $M_{\mathrm{w}}$ and number of protonable amines. As shown in Figure 5a, even the use of relatively high polymer concentrations (w/w 20, $\mathrm{N} / \mathrm{P}=32$ ) displayed no significant increase of luciferase expression compared to untreated cells. One reason might be the inferior condensation properties of plain PAA sequences compared to the fatty acid modified PAAs (Figure 4, 23), as well as the absence of lytic activity in the leakage assay (activity $<10 \%$ at all $\mathrm{pH}$ values). The synergistic effect of polyplex stabilization and lytic activity was reflected in the reporter gene expression profiles of all fatty acid modified polymers. Modification of a K-Stp $2-\mathrm{K}$ sequence with either myristic (9) or oleic acid (10) increased the transfection compared to K$\mathrm{Stp}_{5}-\mathrm{K}(23) 10$-fold at $\mathrm{w} / \mathrm{w} 10(\mathrm{~N} / \mathrm{P}=11)$. Elongation of the chain by two additional Stp units (21 and 22) resulted in an up to 100 -fold increase of reporter gene expression. This was accompanied by a moderate increase in cytotoxicity for the myristic acid derivatives in all tested concentrations (9 and 21). Oleic acid modification had a comparable effect on transfection efficiency, whereas the toxicity of the polymers in the tested concentration range was negligible (10 and 22). Compared to the LPEI control, all polymers showed a 1000-fold lower transfection activity, but demonstrated the beneficial influence of hydrophobic modifications on polyplex stability and gene delivery.

To study the influence of a dimerization handle on transfection efficiency, a single C-terminal cysteine was introduced into i-shape sequences $(69-72)$. The introduction of the C-terminal cysteine into fatty acid bearing polymers showed no significant impact on pDNA complexation, as a w/w of $10(\mathrm{~N} / \mathrm{P}=10-11)$ was still sufficient for complete retardation (see Supporting Information). However, using these dimerizable, fatty acid modified PAAs for pDNA delivery had a significant effect on reporter gene expression. In comparison to their analogous sequences $\mathrm{K}-\mathrm{Stp}_{2}-\mathrm{K}-\mathrm{MyrA}_{2}$ (9) and $\mathrm{K}-\mathrm{Stp}_{2}-\mathrm{K}-\mathrm{OleA}_{2}(\mathbf{1 0})$, the cysteine containing polymers (69 and 71) demonstrated a 100-fold increased reporter gene expression at a $\mathrm{w} / \mathrm{w}$ of $10(\mathrm{~N} / \mathrm{P}=10-11)$. To evaluate the possible influence of the primary amine of the lysine residue on pDNA delivery, sequences without the lysine were screened. Whereas the lysine had no significant effect on the oleic acid modified PAAs (72 vs 71), the delivery efficiency of 69 compared to $\mathbf{7 0}$ was increased 100 -fold. Oleic acid modification had no pronounced effect on cell viability, regardless of sequence composition. C-terminal cysteine modification seemed to be a viable option to increase the delivery capability of short-chain PAAs, a theory which was additionally supported by the results of DLS-measurements (Figure 4), demonstrating the increased stability of cysteine containing polyplexes. These results supported the hypothesis that PAA polymers without the ability of disulfide bridge formation suffer from inadequate stabilization of the resulting polyplexes.

By insertion of a second cysteine into the sequence, the polymers gained the ability of in situ polymerization, allowing the formation of larger polymeric structures. Gel retardation assay (see Supporting Information) showed that in comparison with single cysteine modified PAAs the pDNA complexation was improved, but still heavily dependent on hydrophobic modifications. Figure $5 b(45,46,51)$ showed the reporter gene expression of the cross-linking i-shape formulations with 


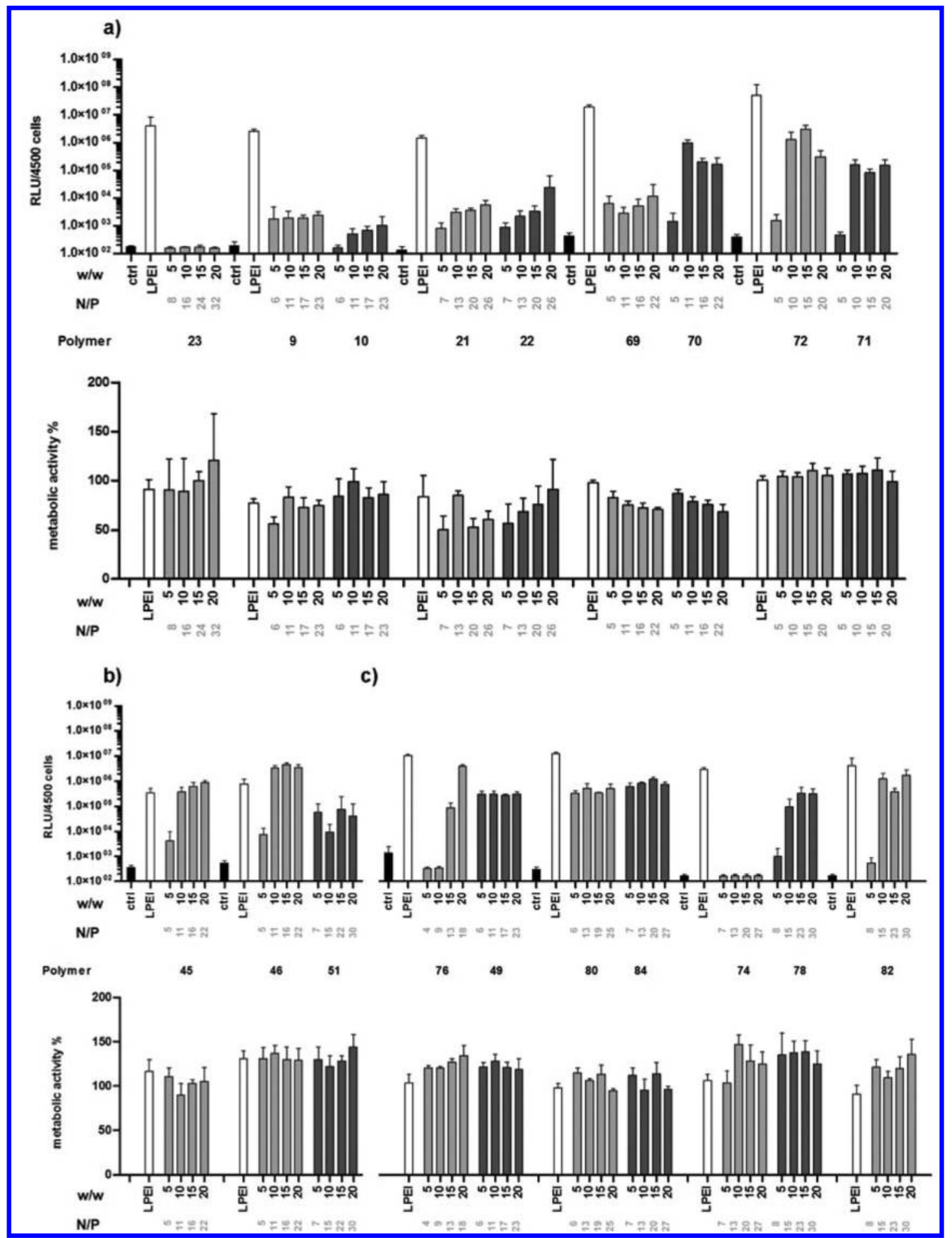

Figure 5. Luciferase reporter gene expression and metabolic activity in Neuro2a cells $24 \mathrm{~h}$ after transfection. Polyplexes were formed at different w/w ratios (polymer/DNA); the LPEI control was always used at its optimal $\mathrm{w} / \mathrm{w}$ of $0.8(\mathrm{~N} / \mathrm{P}=6)$.

different fatty acid modifications on a C-Stp 3 -C-K backbone. The polymers were able to effectively transfer pDNA into target cells and showed a transgene expression profile characterized by a plateau phase beginning at $\mathrm{w} / \mathrm{w} 10(\mathrm{~N} / \mathrm{P}=11-15)$. As soon as this concentration was reached, reporter gene expression was not increased by higher polymer concentrations. For other polymeric delivery systems, this effect was normally connected to increased cytotoxicity at higher polymer concentrations, resulting in reduced transfection levels. In the case of the PAA systems, the MTT assay did not show toxic side effects, indicating additional intracellular delivery bottlenecks. The myristic and oleic acid modified i-shapes were able to compete with the LPEI control in terms of luciferase expression, demonstrating the delivery potential of these structures. The use of the backbone control polymer (51) resulted in a 10- to 50 -fold lower efficiency, highlighting the importance of the hydrophobic modification.

By synthesizing a set of symmetrical polymers with a fixed, central hydrophobic domain (T-shapes), the influence of parameters, like the amount of protonable groups per molecule 
and hydrophilic-lipophilic ratio, were studied. All tested polymers were modified with a dual oleic acid motif at the central lysine, as oleic acid modification was the most effective modification in terms of toxicity and efficiency in previous experiments. Figure 5c (compounds 49-84) shows a comparison between dioleoyl modified T-shapes (76, 49, 80, 84) and not hydrophobically modified linear chains with terminal cysteines $(\mathbf{7 4}, \mathbf{7 8}, \mathbf{8 2})$. The oleoyl T-shape/pDNA complexes were strong enough to prevent migration in the gel at a $\mathrm{w} / \mathrm{w}$ of $10(\mathrm{~N} / \mathrm{P}=9-13)$, whereas the interaction of the unmodified chains at the same $\mathrm{w} / \mathrm{w}(\mathrm{N} / \mathrm{P}=13-15)$ was not strong enough to prevent NA migration (see Supporting Information). Figure 5c shows the transfection efficiency and cytotoxic potential of oleoyl T-shapes with different numbers of Stp building blocks per molecule. The balance between hydrophobic and cationic domain had a significant impact on efficacy, as seen by comparing the transfection efficiency of 49 and 76. Whereas increasing concentrations of 76 led to reporter gene expression almost comparable with the LPEI control, the use of a polymer containing two additional Stp units resulted in an early, only moderate, plateau of activity. The introduction of additional Stp units did not improve their overall performance, regardless of tested concentrations. The reporter gene expression was always 10-fold lower than the LPEI control. These results indicated a fine balance between hydrophobicity and hydrophilicity as exemplified by 76 . Compound 76 had the highest molar proportion of fatty acid substitution in the screen and exhibited the strongest activity in terms of expression level. All other T-shape derivatives could not compete in terms of expression level and reached their maximum level at lower concentrations of $\mathrm{w} / \mathrm{w} 5(\mathrm{~N} / \mathrm{P}=6-7)$.

To verify the importance of the hydrophobic domain and to study the influence of polymer chain length on the efficiency of the T-shape polymers in more detail, a second set of polymers with increasing number of monomer units and without hydrophobic modification (but with cross-linking cysteines) was synthesized and screened $(\mathbf{7 4}, \mathbf{7 8}, \mathbf{8 2})$. Here, the trend of chain length influence was reversed. Compound 74, a structural analogue of $\mathbf{7 6}$, did not show any reporter gene expression, whereas an increase in Stp-building blocks per molecule did result in an increasing gene expression $(\mathbf{7 8}, \mathbf{8 2})$. Compared to the gene expression levels of the oleoyl T-shapes, these polymers had the disadvantage of requiring rather high polymer concentrations to achieve comparable transfection efficiency. The dual fatty acid motif could increase the delivery efficiency, but the influence of the modification diminished with an increasing number of Stp units in the backbone. This supports earlier findings that the hydrophobic modification was more effective on smaller PAAs and could increase their efficiency dramatically, whereas an increasing amount of Stp units in a fatty acid-free polymer also resulted in an improved, but still less efficient delivery.

\section{CONCLUSIONS}

In this study, we successfully designed and synthesized a small library of sequence-defined polymers with optimized $\mathrm{pH}$ dependent lytic activity, pDNA binding, and bioresponsive cargo release. Using a solid-phase assisted synthesis approach and a small set of different modules, we were able to construct sequence-defined, multifunctional polymers. Despite the small size $\left(M_{\mathrm{w}} 500-3100 \mathrm{Da}\right)$ of the polymers, the library screen identified several polymers with a pDNA transfection efficiency comparable to LPEI. The polymers and the synthetic approach could be seen as model systems to increase the knowledge about the rational design of polymeric nonviral vectors and will ultimately lead to even more sophisticated, programmable polymeric vectors.

\section{ASSOCIATED CONTENT}

\section{S Supporting Information}

Additional gel shift data for polymers $(49,69,70,71,72,74$, $76,78,80,82,84)$ and analytical data of all polymers. This material is available free of charge via the Internet at http:// pubs.acs.org.

\section{AUTHOR INFORMATION}

\section{Corresponding Author}

*Phone: ++49-89-2180-77840. Fax: ++49-89-2180-77798. Email: davidsch@mb.au.dk, ernst.wagner@cup.uni-muenchen.de.

Present Address

"Department of Molecular Biology, Århus University, C.F. Møllers Allé, Building 1130, DK-8000 Århus C, Denmark

\section{Notes}

The authors declare no competing financial interest.

\section{Abbreviations:}

ButA, Butyric Acid; CapA, Caprylic Acid; DCM, Dichloromethane; DMF, Dimethylformamide; DMSO, Diemethylsulfoxide; DLS, Dynamic Light Scattering; EtOH, Ethanol; FA, Fatty acid; HBG, HEPES-buffered glucose; HOBt, Hydroxybenzotriazol; LAR, Luciferase assay reagent; $\mathrm{MeOH}$, Methanol; MTT, (3-(4,5-Dimethylthiazol-2-yl)-2,5-diphenyltetrazolium; MyrA, Myristic acid; NA, Nucleic acid; N/P, Nitrogen to Phosphate ratio; OleA, Oleic acid; PAA, Polyaminoamide; PAMAM, Polyamidoamine; PBS, Phosphate-buffered saline; pDNA, plasmid DNA; PEI, Polyethylenimine; RLU, relative light units; siRNA, Small interfering RNA; TBE, Tris-BorateEDTA buffer; TFA, Trifluoroacetic acid; THF, Tetrahydrofurane

\section{ACKNOWLEDGMENTS}

This work was supported by the DFG Cluster "Nanosystems Initiative Munich", a grant from Roche, and the Biotech Cluster $\mathrm{m} 4$ project T12. We want to thank Matthias Wassenberg and Verena Brand for their technical assistance with the in vitro assays and Dr. Lars Allmendinger for helpful discussions about NMR techniques.

\section{REFERENCES}

(1) Friedmann, T., and Roblin, R. (1972) Gene therapy for human genetic disease? Science 175, 949-955.

(2) Ashtari, M., Cyckowski, L. L., Monroe, J. F., Marshall, K. A., Chung, D. C., Auricchio, A., Simonelli, F., Leroy, B. P., Maguire, A. M., Shindler, K. S., and Bennett, J. (2011) The human visual cortex responds to gene therapy-mediated recovery of retinal function. J. Clin. Invest. 121, 2160-8.

(3) Edelstein, M. L., Abedi, M. R., and Wixon, J. (2007) Gene therapy clinical trials worldwide to 2007--an update. J.Gene Med. 9, $833-842$.

(4) Cavazzana-Calvo, M., and Fischer, A. (2007) Gene therapy for severe combined immunodeficiency: are we there yet? J. Clin. Invest. $117,1456-1465$.

(5) Escors, D., and Breckpot, K. (2010) Lentiviral vectors in gene therapy: their current status and future potential. Arch. Immunol. Ther. Exp. (Warsz.) 58, 107-19.

(6) Nair, V. (2008) Retrovirus-induced oncogenesis and safety of retroviral vectors. Curr. Opin. Mol. Ther. 10, 431-8. 
(7) Wiethoff, C. M., and Middaugh, C. R. (2003) Barriers to nonviral gene delivery. J. Pharm. Sci. 92, 203-217.

(8) Mercer, J., Schelhaas, M., and Helenius, A. (2010) Virus entry by endocytosis. Annu. Rev. Biochem. 79, 803-33.

(9) Doms, R. W., Helenius, A., and White, J. (1985) Membrane fusion activity of the influenza virus hemagglutinin. The low $\mathrm{pH}$ induced conformational change. J. Biol. Chem. 260, 2973-81.

(10) Behr, J. P. (1997) The proton sponge: A trick to enter cells the viruses did not exploit. Chimia 51, 34-36.

(11) Ogris, M., Carlisle, R. C., Bettinger, T., and Seymour, L. W. (2001) Melittin enables efficient vesicular escape and enhanced nuclear access of nonviral gene delivery vectors. J. Biol. Chem. 276, 47550-47555.

(12) Subbarao, N. K., Parente, R. A., Szoka, F. C., Jr., Nadasdi, L., and Pongracz, K. (1987) pH-dependent bilayer destabilization by an amphipathic peptide. Biochemistry 26, 2964-2972.

(13) Oskuee, R. K., Dehshahri, A., Shier, W. T., and Ramezani, M. (2009) Alkylcarboxylate grafting to polyethylenimine: a simple approach to producing a DNA nanocarrier with low toxicity. J. Gene Med. 11, 921-32.

(14) Philipp, A., Zhao, X., Tarcha, P., Wagner, E., and Zintchenko, A. (2009) Hydrophobically modified oligoethylenimines as highly efficient transfection agents for siRNA delivery. Bioconjugate Chem. 20, 2055-61.

(15) Barz, M., Tarantola, M., Fischer, K., Schmidt, M., Luxenhofer, R., Janshoff, A., Theato, P., and Zentel, R. (2008) From defined reactive diblock copolymers to functional HPMA-based self-assembled nanoaggregates. Biomacromolecules 9, 3114-8.

(16) Allmeroth, M., Moderegger, D., Biesalski, B., Koynov, K., Rosch, F., Thews, O., and Zentel, R. (2011) Modifying the body distribution of HPMA-based copolymers by molecular weight and aggregate formation. Biomacromolecules 12, 2841-9.

(17) Rozema, D. B., Lewis, D. L., Wakefield, D. H., Wong, S. C., Klein, J. J., Roesch, P. L., Bertin, S. L., Reppen, T. W., Chu, Q., Blokhin, A. V., Hagstrom, J. E., and Wolff, J. A. (2007) Dynamic PolyConjugates for targeted in vivo delivery of siRNA to hepatocytes. Proc. Natl. Acad. Sci. U. S. A. 104, 12982-12987.

(18) Hartmann, L., Krause, E., Antonietti, M., and Borner, H. G. (2006) Solid-phase supported polymer synthesis of sequence-defined, multifunctional poly(amidoamines). Biomacromolecules 7, 1239-1244.

(19) Schaffert, D., Badgujar, N., and Wagner, E. (2011) Novel Fmocpolyamino acids for solid-phase synthesis of defined polyamidoamines. Org. Lett. 13, 1586-9.

(20) Schaffert, D., Troiber, C., Salcher, E. E., Frohlich, T., Martin, I., Badgujar, N., Dohmen, C., Edinger, D., Klager, R., Maiwald, G., Farkasova, K., Seeber, S., Jahn-Hofmann, K., Hadwiger, P., and Wagner, E. (2011) Solid-Phase Synthesis of Sequence-Defined T-, i-, and U-Shape Polymers for pDNA and siRNA Delivery. Angew. Chem., Int. Ed. Engl. 50, 8986-8989.

(21) Schaffert, D., Kiss, M., Rodl, W., Shir, A., Levitzki, A., Ogris, M., and Wagner, E. (2011) Poly(I:C)-mediated tumor growth suppression in EGF-receptor overexpressing tumors using EGF-polyethylene glycol-linear polyethylenimine as carrier. Pharm. Res. 28, 731-41.

(22) Mosmann, T. (1983) Rapid colorimetric assay for cellular growth and survival: Application to proliferation and cytotoxicity assays. J. Immunol. Methods 65, 55-63.

(23) Wang, Y., Chen, P., and Shen, J. (2006) The development and characterization of a glutathione-sensitive cross-linked polyethylenimine gene vector. Biomaterials 27, 5292-5298.

(24) Lee, R. J., Wang, S., and Low, P. S. (1996) Measurement of endosome $\mathrm{pH}$ following folate receptor-mediated endocytosis. Biochim. Biophys. Acta 1312, 237-242.

(25) Felgner, P. L., Gadek, T. R., Holm, M., Roman, R., Chan, H. W., Wenz, M., Northrop, J. P., Ringold, G. M., and Danielsen, M. (1987) Lipofection: A highly efficient, lipid mediated DNA-transfection procedure. Proc. Natl. Acad. Sci. U. S. A. 84, 7413-7417. 